\title{
Cokrigagem de razões logaritmicas aditivas (alr) na estimativa de teores em depósitos de ferro
}

\section{Cokriging of additive log-ratios (alr) for grade estimation in iron ore deposits}

\begin{abstract}
Maria Noel Morales Boezio
Eng. Química, MSc, PhD

Universidade Federal do Rio Grande do Sul. maria.morales@ufrgs.br

\section{João Felipe Coimbra Leite Costa \\ Eng. de Minas, MSc, PhD, \\ Universidade Federal do Rio Grande do Sul. \\ jfelipe@ufrgs.br}

\section{Jair Carlos Koppe}

Geólogo, Eng. de Minas, MSc, DSc,

Universidade Federal do Rio Grande do Sul. jkoppe@ufrgs.br

\section{Resumo}

Os produtos do minério de ferro são definidos pelos teores de ferro e dos contaminantes e, também, pelas partições granulométricas. Os dados que provêm dos depósitos de ferro constituem dados composicionais, sendo um vetor de variáveis com uma soma fechada dada pelos balanços de cada espécie, nas diversas frações granulométricas, ou pelas espécies em cada fração granulométrica. As somas fechadas conduzem a correlações espúrias e, uma condição chamada de viés negativo. Essa condição impede as estimativas de satisfazerem os balanços e possibilita que algumas estimativas sejam negativas ou estejam fora do intervalo de valores dos dados originais. As metodologias clássicas da geoestatística não levam em consideração esses fatos e as práticas comuns utilizam alternativas que forçam o fechamento das somas, tais como a utilização do modelo de corregionalizacão intrínseca, a determinação de uma variável por diferença ou a distribuição do erro, sendo que, em estimativas negativas, estas devem ser substituídas por valores válidos. Nesse artigo, apresenta-se a cokrigagem de razões logarítmicas aditivas $(a l r)$, transformação desenvolvida para dados composicionais, como uma metodologia alternativa para se determinarem teores em minérios de ferro, onde se tem múltiplas variáveis correlacionadas com somas constantes. Obtêm-se melhores resultados do que por cokrigagem dos dados originais, com todas as estimativas no intervalo original das amostras, satisfazendo-se as somas fechadas consideradas, sem necessidade de pós-processamento.

Palavras-chave:Dadoscomposicionais, cokrigagem, minériodeferro, corregionalização.

\begin{abstract}
Iron ore products are defined by their iron and contaminant grades and also by the granulometric partitions. Data from iron ore deposits constitute compositional data, involving a vector of variables adding up to a constant sum given by the mass balance among granulometric partitions or among chemical species in each granulometric fraction. The closed sums lead to spurious correlations and to a negative bias condition. This condition leads to estimates that do not satisfy the balances and estimates that take negative values or do not belong to the interval of values of the original data. Classic geostatistical methodologies do not take these facts into account and the common practices force the sum through determining one variable by difference, distributing the sum error or using an intrinsic corregionalization model and substituting of the negative values by valid ones. In this paper, cokriging of additive log-ratios (alr), a transformation developed for compositional data, is presented as an alternative methodology to estimate grades in iron ores, in presence of multiple correlated variables with a closed sum. Results obtained through this methodology are better than the ones obtained by direct cokriging of the original data, leading to positive estimates, all in the original data interval and satisfying the considered constant sums, without post-processing.
\end{abstract}

Keywords: Compositional data, cokriging, iron ore, corregionalization. 


\section{Introdução}

Os produtos provenientes do minério de ferro são caracterizados pelos teores de ferro e dos contaminantes que interferem no processo siderúrgico e pela sua granulometria. Múltiplas variáveis correlacionadas precisam de ser determinadas de forma simultânea. A metodologia clássica, mais apropriada para satisfazer esse propósito, é a cokrigagem (Marechal, 1970).

No caso do ferro e da sílica presentes nos itabiritos, a correlação inversa existente pode ser explicada pela gênese do depósito. No entanto, os dados que provêm dos depósitos de ferro constituem dados composicionais: um vetor de

A

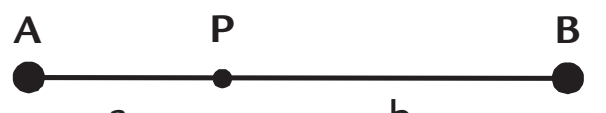

a

b

$$
\mathrm{a}+\mathrm{b}=\mathrm{K}
$$

Aitchison (1981) foi o primeiro a introduzir a idéia de que os dados composicionais só carregam informação relativa e não absoluta do valor das componentes. Portanto a informação é mais bem expressa em termos de quocientes (razões) ou em termos de razões logarítmicas, já que as mesmas são de mais fácil manipulação matemática. A vantagem da transformação em razões logarítmicas é que tal medida leva os dados do espaço amostral restrito (simplex) ao espaço real irrestrito e multivariado.

A transformação em razões logarítmicas é uma transformação não linear de razões logarítmicas e não das variáveis originais, pelo que não seria esperada a introdução de um viés pela transformação não linear. Nesse sentido, PawlowskyGlahn e Egozcue (2002) apresentam uma abordagem geométrica, introduzindo os conceitos de variância métrica e estimador composicional não tendencioso. $\mathrm{O}$ estimador "c-BLU" (do inglês best linear compositional estimator) é o melhor estimador linear composicional, não tendencioso, com respeito à geometria do simplex - ao centro da distribuição de uma composição randômica, que é a média geométrica. variáveis com uma soma constante, dada pelos balanços de massa de cada espécie presente em várias faixas granulométricas e o balanço de massa das espécies envolvidas em cada faixa. A correlação espacial existente é afetada pela soma e pode ser considerada espúria (Pearson, 1897; Pawlowsky-Glahn \& Olea, 2004).

O espaço amostral ao qual pertencem os dados composicionais não é o espaço real. Os dados composicionais estão restringidos a um espaço amostral chamado D-simplex, de dimensão D-1, sendo $\mathrm{D}$ o número de componentes que satisfazem a soma fechada. $\mathrm{O}$ conceito de simplex pertence ao campo da álge-

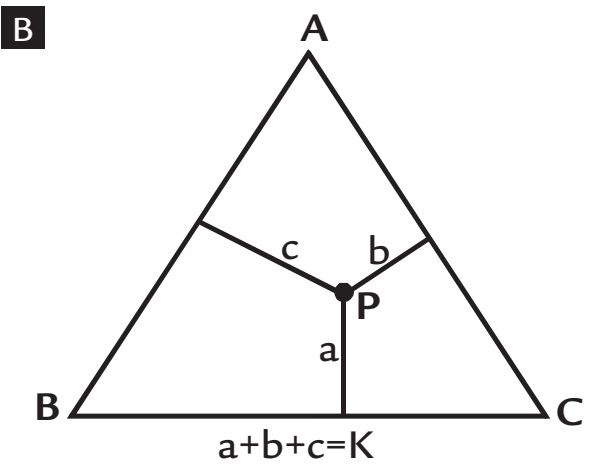

bra topológica, sendo a generalização de regiões triangulares $(D=3)$ ou tetraédricas $(\mathrm{D}=4)$ ), para $n$ dimensões (Munkres, 1993).

No caso de uma composição com duas componentes, A e B, com soma constante $\mathrm{K}$, o D simplex (2-simplex) é um segmento de reta de dimensão D-1=1, no qual a soma constante $\mathrm{K}$ é válida em cada ponto $\mathrm{P}$ da mesma (Figura 1A). $\mathrm{Na}$ presença de 3 componentes, A, B e $\mathrm{C}$, também, com soma constante $\mathrm{K}$, o 3-simplex é um triângulo eqüilátero de duas dimensões (D-1), em que qualquer ponto $\mathrm{P}$ satisfaz a soma constante $\mathrm{K}$ ( $\mathrm{Fi}$ gura $1 \mathrm{~B})$.

Figura 1

Representação gráfica do simplex (espaço amostral) correspondente a duas composições:

(A) de duas componentes $A$ e $B$.

(B) de três componentes $\mathrm{A}, \mathrm{B}$ e $\mathrm{C}$, com soma constante $\mathrm{K}$.

Esses conceitos foram estendidos a dados regionalizados (Matheron, 1964) por Pawlowsky-Glahn e Olea (2004) e Tolosana-Delgado (2006). Tais autores alertaram para o fato de que a matriz de covariâncias cruzadas, no caso de dados composicionais, é singular, não permitindo a cokrigagem direta dos dados originais. Também, demonstra-se, na presença de dados composicionais, a existência de um viés negativo (Pawlowsky-Glahn \& Olea, 2004), que é introduzido ao se considerarem as covariâncias cruzadas e diretas necessárias para a modelagem da corregionalização necessária na cokrigagem. Portanto a presença desse viés faz com que a cokrigagem já não forneça um estimador não tendencioso, no caso de dados composicionais.

$\mathrm{Na}$ determinação de teores em depósitos de ferro, um dos aspectos que interessam é a reprodução das somas constantes originais. Para esse problema, são apresentadas duas alternativas (Goovaerts, 1997): (i) deixar uma variável fora do sistema e determiná-la numa etapa posterior; (ii) determinar todas as variáveis e distribuir o erro de fechamento para satisfazer a soma constante. Outra alternativa é utilizar um modelo de cor- regionalização intrínseco (Wackernagel, 1994; Chilès \& Delfiner, 1999) e, dessa forma, outorgar o mesmo peso a todos os dados de todas as variáveis presentes, tendo em vista que esse modelo assume que a correlação espacial é independente do vetor de separação $h$. Nesse contexto, a cokrigagem fornece os mesmos resultados que a krigagem individual (Wackernagel, 1994). Porém a hipótese das variáveis estarem em correlação intrínseca não é, necessariamente, apropriada se não for satisfeita na prática e, sim, imposta na modelagem.

Outro fato a ser considerado é a presença de estimativas negativas ou fora do intervalo original das amostras, dada pela condição de viés negativo (Pawlowsky-Glahn \& Olea, 2004), pela correlação espúria e pelas matrizes singulares de covariância cruzada. Geralmente, es $\neg$ ses valores inadequados são substituídos por valores válidos: metade do limite de detecçao, mínimo da distribuiçao original, médias locais, etc., numa etapa de pós-processamento.

Esse artigo propõe a cokrigagem de razões logarítmicas aditivas como uma metodologia alternativa para determinar múltiplas variáveis correlacionadas, que 
satisfaçam uma soma fechada. A metodologia é apresentada num estudo de caso proveniente do Quadrilátero Ferrífero, no estado de Minas Gerais, com objetivo de se determinarem os teores de ferro e dos contaminantes, assim como a massa das frações granulométricas de interesse. Os resultados são comparados com os obtidos por cokrigagem dos dados originais.

\section{Metodologia}

A metodologia proposta é a cokrigagem das razões logarítmicas aditivas $(a l r)$, metodologia alternativa para se determinarem teores em minérios de ferro, onde existam múltiplas variáveis correlacionadas que satisfaçam somas constantes.
A metodologia pode ser revisada de forma extensa em Pawlowsky-Glahn e Egozcue (2001), Egozcue et al. (2003) e em Pawlowsky-Glahn e Olea (2004).

Uma composição regionalizada de dimensão $\mathrm{D}$ é um vetor randômico $\mathrm{Z}(u)=\left(Z_{1}(u), Z_{2}(u), \ldots, Z_{D}(u)\right)^{T} \operatorname{com} D$ componentes e que tem uma soma constante $c$ e satisfaz as seguintes condições:

i) Todas as componentes são estritamente positivas $Z_{i}(u)>0$, $i=1,2, \ldots, D$.

ii) A soma de todas as componentes é constante $c$ :

$$
\sum_{i=1}^{D} Z_{i}(u)=c \quad \text { se } c=1: \sum_{i=1}^{D} Z_{i}(u)=j^{\top} Z(u)=1 \quad j=(1,1, \ldots, 1)^{\top}
$$

iii) O espaço amostral de $\mathbf{Z}(u)$ é o $\mathrm{D}$-simplex $S^{D}$, espaço de (D-1) dimensões, no qual é satisfeita a soma constante $c$ :

$$
S^{D}=\left\{Z(u) ; Z_{i}(u)>0, i=1,2, \ldots D ; \sum_{i=1}^{D} Z_{i}(u)=c\right\}
$$

Apesar de a razão logarítmica aditiva (alr) não preservar a métrica do simplex, ela é mais simples de se usar do que a razão logarítmica isométrica $(i l r)$. Já a razão logarítmica centrada $(c l r)$ não pode ser utilizada, porque se constitui em uma matriz de covariâncias singular. A metodologia proposta, também, diminui em uma unidade o número de variáveis que intervém na modelagem do modelo linear de corregionalização (MLC) (Wackernagel, 1994).

A razão logarítmica aditiva é definida como:

alr $: S^{D} \rightarrow R^{D-1}$

$$
Z(u) \rightarrow \operatorname{alr}(Z(u))=Y(u)=F \cdot \ln (Z(u)) \quad F=(I:-j)=\left(\begin{array}{ccccc}
1 & 0 & \ldots & 0 & -1 \\
0 & 1 & \ldots & 0 & -1 \\
\vdots & \vdots & \ddots & \vdots & \vdots \\
0 & 0 & \ldots & 1 & -1
\end{array}\right)
$$

onde I é a matriz identidade de dimensões (D-1)x(D-1) e ln é o logaritmo natural.

Para cada componente, a transfor- mação é obtida dividindo-se a variável original pela componente $D$ e computando-se o logaritmo natural, obtendo o

vetor $\mathrm{Y}(u)$ com $(\mathrm{D}-1)$ componentes:

O sistema na equação (4) pode ser cokrigado e retrotransformado ao espaço original (o simplex), utilizando- se a transformação logística aditiva generalizada $(a g l)$, cuja definição inclui o vetor auxiliar X $(u)$ (Pawlowsky-Glahn and Olea, 2004; Tolosana-Delgado, 2006):

$$
\begin{gathered}
\left.X(u)=Y_{1}(u) Y_{2}(u) \ldots Y_{D-1}(u) 0\right)^{T} \\
\text { agl: } R^{D-1} \rightarrow S^{D} Z(u)=c \cdot \operatorname{ag}(Y(u)) \\
Y(u) \rightarrow Z(u)=\frac{\exp (X(u))}{j^{T} \operatorname{ex}(X(u)} \\
\exp \left(X(u)=\left(\operatorname { e x p } \left(Y _ { 1 } ( u ) \operatorname { e x p } \left(Y_{2}(u) \ldots \exp \left(Y_{D-1}(u) 1\right)^{T}\right.\right.\right.\right.
\end{gathered}
$$

Ao se utilizar essa transformação, pela sua construção matemática, é garantido o fechamento das somas correspondentes aos balanços de massa entre espécies químicas para cada faixa granulométrica e global. A cokrigagem das razões logarítmicas aditivas é recomendada no caso em que esses fechamentos precisem ser reproduzidos nos modelos estimados. No estudo de caso, apresentase a metodologia numa sequência passo a passo.

\section{Estudo de caso}

O estudo de caso provém da Mina de Sapecado, pertencente à VALE, localizada no Quadrilátero Ferrífero, no Com- plexo Pico. O banco de dados e modelos geológicos estão expressos em coordenadas locais, alinhadas, com a direção prin- cipal do corpo de minério.

Vários tipos de itabiritos de importância econômica podem ser agrupados 
num só domínio geoestatístico chamado de IB, com teores de ferro entre 30 e $64 \%$. Os dados foram regularizados em $10 \mathrm{~m}$, com um espaçamento amostral médio no plano XY desde $50 \times 50 \mathrm{~m}$ até $200 \times 100$ $\mathrm{m}$ nas direções $\mathrm{X}$ e $\mathrm{Y}$, respectivamente. A configuração dos dados é isotópica (Wackernagel, 1994), com todas as variáveis presentes em todos os locais amostrais.

A granulometria permite caracterizar três tipos de produtos: granulado (fração 1), sinter feed (fração 2) e pellet feed (fração 3). As amostras foram analisadas em cada fração granulométrica e no global.

Os atributos de interesse são a massa retida da fração granulométrica, os teores de ferro, de alumina, de sílica, de fósforo e de manganês e as perdas por calcinação, denominadas, respectivamente, Wi, FEi, ALi, SIi, Pi, MNi e PPCi, onde o índice i corresponde-se com as frações granulométricas $\mathrm{i}=1,2,3$ e global $(\mathrm{T})$.

Para se levarem em consideração as diferenças de suporte, os teores são acumulados pela massa de cada fração. O teor de ferro é expresso como complementar ao valor máximo em hematita (70\%), para se ter uma correlação positiva com o teor de sílica.

As 27 variáveis a serem determinadas estão relacionadas por balanços, de massa que devem estar expressos como somas constantes. Na Figura 2, são apresentadas essas somas constantes, as quais aparecem numeradas, para serem refe- renciadas na implementação da metodologia passo a passo.

A sequência de cálculo apresentada na Figura 2, para esse estudo de caso, consiste em:

- Estimar, inicialmente, as massas das frações granulométricas nas faixas 1 , 2 e 3, W1Y(u), W2Y(u) e W3Y(u), respectivamente.

- Numa segunda instância, são estimados os quocientes das estimativas de alumina na faixa 1, 2 e 3 sobre o global (ALIY(u)/ALTY(u)), (AL2Y(u)/ $\operatorname{ALTY}(u))$ e (AL3Y(u)/ALTY(u)), já que a concentração global cancela-se nos quocientes e estes devem ser determinados numa etapa posterior.

- Para o manganês, fósforo, perdas por calcinação e sílica, o procedimento é igual ao da alumina.

- Os teores globais são determinados utilizando o balanço de massa correspondente aos teores globais, substituindo-os nos quocientes determinados anteriormente $((\mathrm{ALlY}(\mathrm{u}) /$ $\operatorname{ALTY}(\mathrm{u})), \quad(\operatorname{AL2Y}(\mathrm{u}) / \operatorname{ALTY}(\mathrm{u})) \quad \mathrm{e}$ $(\operatorname{AL3Y}(\mathrm{u}) / \operatorname{ALTY}(\mathrm{u}))$, no caso da alumina) para determinar $\operatorname{AL} 1 Y(u)$, $\operatorname{AL} 2 Y(u)$ e AL3Y(u), respectivamente.

- Procede-se, de igual forma, para o manganês, fósforo, perdas por calcinação e sílica, determinando as estimativas $\mathrm{MN} 1 \mathrm{Y}(\mathrm{u}), \mathrm{MN} 2 \mathrm{Y}(\mathrm{u})$ e $\operatorname{MN3Y}(u), \operatorname{P1Y}(u), P 2 Y(u)$ e P3Y(u), $\operatorname{PPC1Y}(u), \operatorname{PPC} 2 Y(u)$ e $\operatorname{PPC} 3 Y(u)$ e
$\operatorname{SI} 1 Y(u), \operatorname{SI} 2 Y(u)$ e SI3Y(u).

- Estimam-se os teores de ferro nas faixas 1, 2, 3 e global, já que a soma constante do ferro é diferente das anteriores, por causa do ferro estar expresso como complementar ao teor máximo em hematita de $70 \%$.

Observa-se que, no caso dos teores de ferro, os mesmos podem ser determinados em todas as faixas e no global, já que esse último não se cancela no quociente. Mas isso conduz à obtenção de dois teores de ferro global: FETY(u) e FETY2(u). O valor retido é escolhido de forma arbitrária FETY(u). Essa situação é uma particularidade do caso de estudo apresentado, por causa de o teor de ferro estar expresso como complementar ao teor máximo em hematita (70\%).

A sequência apresentada, na Figura 2, poderia ser modificada por outra, utilizando-se as somas constantes em outra ordem ou utilizando os balanços de massa entre espécies em cada fração granulométrica.

As etapas apresentadas a seguir, em cada item da sequência mostrada anteriormente, são referenciadas de forma geral, exemplificadas para o caso das massas nas frações 1, 2 e 3:

1. Seleção da variável que será utilizada no denominador (escolheu-se W3(u) de forma arbitrária).

2. Dividem-se e aplicam-se logaritmos, obtendo-se duas razões logarítmicas $\operatorname{alr}(\mathrm{Y} 1 \mathrm{BW}(\mathrm{u})$ e $\mathrm{Y} 2 \mathrm{BW}(\mathrm{u}))$.

$$
Y_{1 B W}(u)=\ln \left(\frac{W_{1}(u)}{W_{3}(u)}\right) \quad Y_{2 B W}(u)=\ln \left(\frac{W_{1}(u)}{W_{3}(u)}\right)
$$

3. Analisa-se a variabilidade espacial conjunta das duas novas variáveis definidas pela equação (6), obtendo-se as principais direções de anisotropia em $\mathrm{N} 68^{\circ}$ e $\mathrm{N} 158^{\circ}$, no plano XY (horizontal), e a menor perpendicular a esse plano, D- $90^{\circ}$.

4. Modelam-se com o modelo linear de corregionalização (MLC), com um efeito pepita e duas estruturas esféricas, com alcances máximos de 140, 130 e $45 \mathrm{~m}$, nas respectivas direções de anisotropia.

$$
\gamma_{B W}=C_{0}+C_{1} \cdot \operatorname{Sph}\left(\frac{70 m}{N 68^{\circ}} \frac{50 m}{N 158^{\circ}} \frac{20 m}{D-90^{\circ}}\right)+C_{2} \cdot \operatorname{Sph}\left(\frac{140 m}{N 68^{\circ}} \frac{130 m}{N 158^{\circ}} \frac{45 m}{D-90^{\circ}}\right)
$$

5. As matrizes de corregionalização $\mathrm{C}_{0}, \mathrm{C}_{1}$ e $\mathrm{C}_{2}$ são determinadas de modo a se satisfazerem as condições de definição positiva requeridas pelo
MLC (Tabela 1).

6. As estimativas das razões logarítmicas aditivas (alr) $\mathrm{Y}^{*} 1 \mathrm{BW}(\mathrm{u})$ e $\mathrm{Y} * 2 \mathrm{BW}(\mathrm{u})$ são ob-

\begin{tabular}{c|c|c}
\hline \multirow{2}{*}{$\mathrm{C}_{0}$} & Y1BW & Y2BW \\
\cline { 2 - 3 } & 0.14 & 0.03 \\
\cline { 2 - 3 } & 0.03 & 0.01 \\
\hline \multirow{2}{*}{$\mathrm{C}_{1}$} & 0.13 & 0.04 \\
\cline { 2 - 3 } & 0.04 & 0.01 \\
\hline \multirow{2}{*}{$\mathrm{C}_{2}$} & 0.91 & 0.32 \\
\cline { 2 - 3 } & 0.32 & 0.25 \\
\hline
\end{tabular}

tidas por cokrigagem ordinária, utilizando-se a vizinhança e a estratégia de busca apresentada na Tabela 2.

\section{Tabela 1}

Matrizes de corregionalização $C_{0}, C_{1}$ e $C_{2}$ obtidas para Y1BW(u) e Y2BW(u). 
Tabela 2

Vizinhança e estratégia de busca para a cokrigagem ordinária das razões logarítmicas aditivas (alr).

7. Retorna-se ao espaço amostral original (o simplex), utilizando-se a trans-

\section{Discussão de resultados}

$\mathrm{O}$ aspecto mais importante a ser discutido, numa primeira instância, é a não tendenciosidade das estimativas, o que pode ser observado pela reprodução da média global e local. Para avaliar a média global, compara-se o valor das médias das estimativas com as médias dos dados originais desagrupados (Isaaks \& Srivastava, 1989) e com as estimativas obtidas por cokrigagem dos dados originais sem pós-processar (Tabela 3).

A cokrigagem dos dados originais é realizada nos subgrupos de variáveis dados pelos balanços de cada variável entre faixas granulométricas e global, deixando fora dos sistemas de cokrigagem a variável correspondente à faixa 1 , determinando-se, posteriormente, a diferença, a qual irá carregar o somatório dos erros das outras determinações. Os dados negativos ou fora do intervalo original são pós-processados, substituindo-os pelas médias locais.

As médias locais foram analisadas para o ferro, sílica e alumina globais, apresentando as tendências

\begin{tabular}{c|c}
\hline Tipo de vizinhança & Móvel \\
\hline Elipsóide de busca & $\begin{array}{c}\text { Alinhado com as } \\
\text { direções de anisotropia }\end{array}$ \\
\hline Alcance na direção de maior continuidade & $750 \mathrm{~m}$ \\
\hline Alcance na direção intermediária & $220 \mathrm{~m}$ \\
\hline Alcance na direção de menor continuidade & $60 \mathrm{~m}$ \\
\hline Número de setores angulares & 8 \\
\hline Múnimo de amostras primárias e secundárias & 3 \\
\hline Discretização do bloco em X ótimo de amostras primárias e secundárias por setor & 2 \\
\hline Discretização do bloco em Y & 5 \\
\hline Discretização do bloco em Z & 5 \\
\hline
\end{tabular}

formação logística aditiva generaliza$\mathrm{da}(a g l)$, onde $\mathrm{K}_{\mathrm{w}}$ é definido de forma

$$
\begin{gathered}
W_{1 Y}(u)=100 \frac{\exp \left(Y_{1 B W}^{*}(u)\right)}{K_{W}} \\
W_{2 Y}(u)=100 \frac{\exp \left(Y_{2 B W}^{*}(u)\right)}{K_{W}} \\
W_{3 Y}(u)=\frac{100}{K_{W}} \\
K_{W}=1+\exp \left(Y_{1 B W}^{*}(u)\right)+\exp \left(Y_{2 B W}^{*}(u)\right)
\end{gathered}
$$

dos dados originais, das estimativas obtidas por cokrigagem das razões logarítmicas aditivas (alr), e também foram analisadas através da cokrigagem dos dados originais pós-processadas, ao longo das direções das coordenadas X, Y e Z (análises de deriva) (Figura 3). Observa-se que a tendência geral é, aproximadamente, a mesma nos dois casos.

$\mathrm{Na}$ Figura 4, são apresentados os diagramas de dispersão, para as mesmas variáveis, das estimativas obtidas por cokrigagem das razões logarítmicas aditivas (alr) e por cokrigagem dos dados originais pósprocessados. O coeficiente de correlação, entre ambas estimativas, é elevado, mostrando uma dispersão, aproximadamente, simétrica, em torno da bissetriz.

O mapa das estimativas de ferro global obtido pelas duas metodologias é apresentado na Figura 5, para um valor de coordenada $Z=1345 \mathrm{~m}$, onde se observam, também, os dados originais mais próximos a essa seção. Os mapas são semelhantes, com uma melhor reprodução dos valores extremos pela cokrigagem das razões logarítmicas aditivas (alr).

No que se refere à satisfação das somas fechadas, utilizando-se os valores estimados, salienta-se que, ao se utilizar a cokrigagem das razões logarítmicas aditivas $(a l r)$, as somas fechadas são satisfeitas pela totalidade das estimativas, como é observado na Figura 6. Na implementaçao da cokrigagem dos dados originais, os teores da faixa 1 sao determinados por diferença, o que garante o fechamento. Alternativas dessa abordagem, utilizadas para garantir o fechamento das somas na cokrigagem dos dados originais, são a distribuição do erro, ou a utilização do modelo de correlação intrínseca $\mathrm{MCI}$ em vez do modelo linear de corregionalização MLC.

Os balanços entre espécies químicas, para cada faixa granulométrica e global, os quais são conferidos numa etapa posterior, são adequados em ambos os casos (Figura 7). Não é possível satisfazer, simultaneamente, os balanços de massa para a mesma espécie entre faixas e os balanços de massa entre espécies na mesma faixa. 


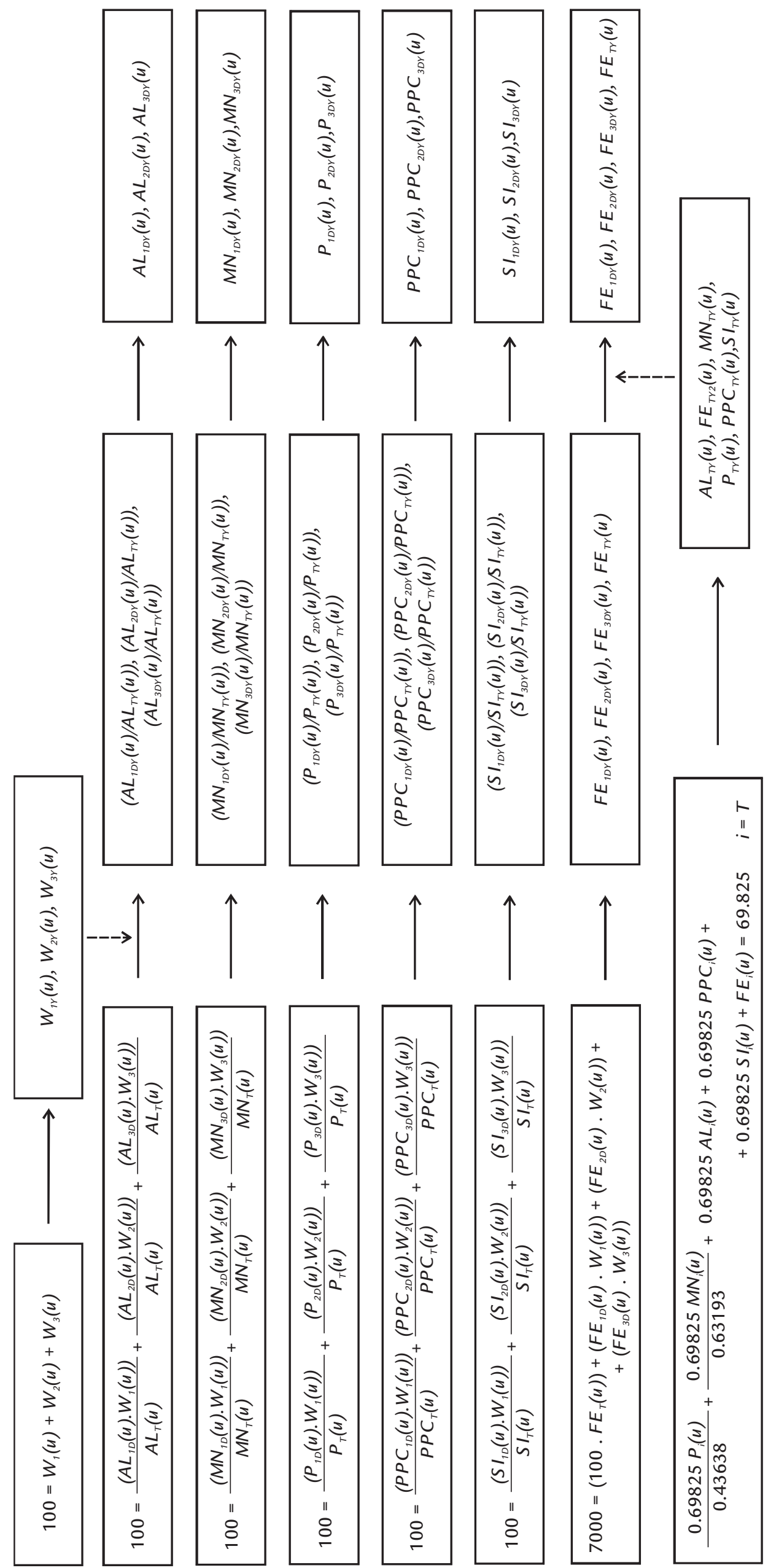

Figura 2

Esquema da sequência de cálculo com somas constantes. O subíndice D corresponde aos dados sem acumular massa (desacumulados) e o subíndice $Y$ corresponde às variáveis estimadas. 

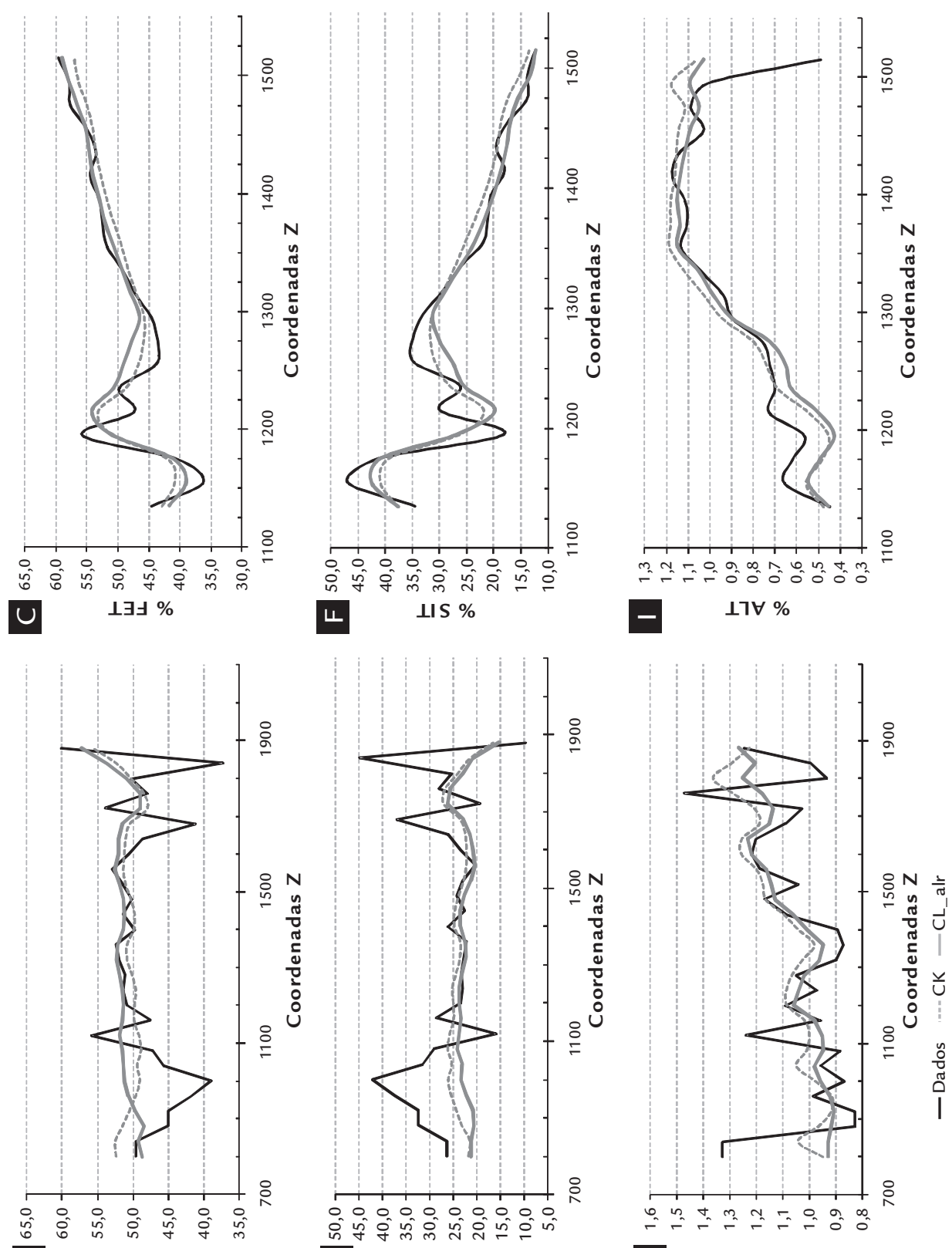

$\infty$

ш

LIS \%

I $\quad \perp 7 \forall \%$

Figura 3 Tendência dos dados originais, estimativas obtidas por cokrigagem direta dos dados originais (CK) e cokrigagem das razões logarítmicas aditivas (CK_alr), ao longo das coordenadas $\mathrm{X}, \mathrm{Y}$ e Z, para os teores globais de ferro $((A),(B)$ e $(C))$, sílica $((D),(E)$ e $(F))$ e alumina $((G),(H)$ e (I)), respectivamente.

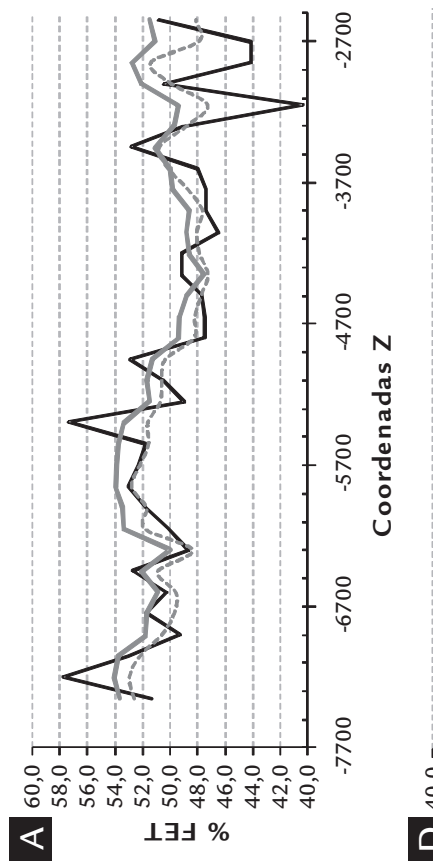

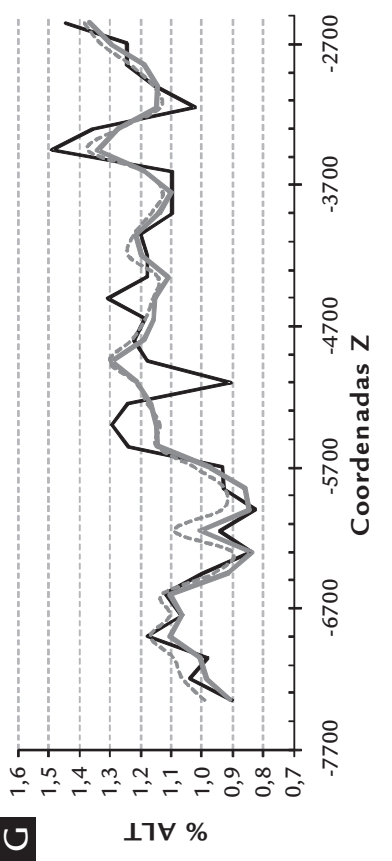




\begin{tabular}{|c|c|c|c|c|}
\hline & \multirow{2}{*}{\begin{tabular}{|c|} 
Dados originais \\
Média desagrupada
\end{tabular}} & \multicolumn{3}{|c|}{ Estimativas obtidas por cokrigagem } \\
\hline & & Média CK_alr & $\begin{array}{l}\text { Média CK sem } \\
\text { pós-processar }\end{array}$ & $\begin{array}{l}\text { Número de blocos } \\
\text { pós-processados CK }\end{array}$ \\
\hline AL1 & 0,86 & 0,84 & 0,87 & 30 \\
\hline AL2 & 0,71 & 0,74 & 0,77 & 3 \\
\hline AL3 & 1,34 & 1,33 & 1,38 & 0 \\
\hline ALT & 1,05 & 1,07 & 1,11 & 0 \\
\hline FE1 & 58,63 & 60,97 & 60,19 & 20 \\
\hline FE2 & 53,44 & 55,61 & 54,28 & 4 \\
\hline FE3 & 43,99 & 46,63 & 44,88 & 0 \\
\hline FET & 49,46 & 51,64 & 50,33 & 0 \\
\hline MN1 & 0,19 & 0,10 & 0,23 & 603 \\
\hline MN2 & 0,22 & 0,13 & 0,26 & 825 \\
\hline MN3 & 0,13 & 0,10 & 0,14 & 332 \\
\hline MNT & 0,17 & 0,11 & 0,19 & 448 \\
\hline P1 & 0,06 & 0,06 & 0,06 & 19 \\
\hline P2 & 0,05 & 0,05 & 0,05 & 0 \\
\hline P3 & 0,05 & 0,05 & 0,05 & 11 \\
\hline PT & 0,05 & 0,05 & 0,05 & 0 \\
\hline PPC1 & 2,69 & 2,79 & 2,89 & 89 \\
\hline PPC2 & 2,00 & 2,13 & 2,24 & 0 \\
\hline PPC3 & 1,95 & 2,15 & 2,14 & 8 \\
\hline РPCT & 2,04 & 2,22 & 2,28 & 0 \\
\hline SI1 & 10,96 & 8,06 & 9,70 & 48 \\
\hline $\mathrm{S} 12$ & 18,43 & 17,05 & 18,76 & 5 \\
\hline $\mathrm{SI} 3$ & 32,18 & 30,14 & 31,74 & 0 \\
\hline SIT & 24,02 & 22,69 & 24,06 & 0 \\
\hline W1 & 17,71 & 16,02 & 17,85 & 0 \\
\hline W2 & 28,08 & 29,16 & 28,28 & 0 \\
\hline W3 & 54,21 & 54,82 & 53,87 & 0 \\
\hline
\end{tabular}

A

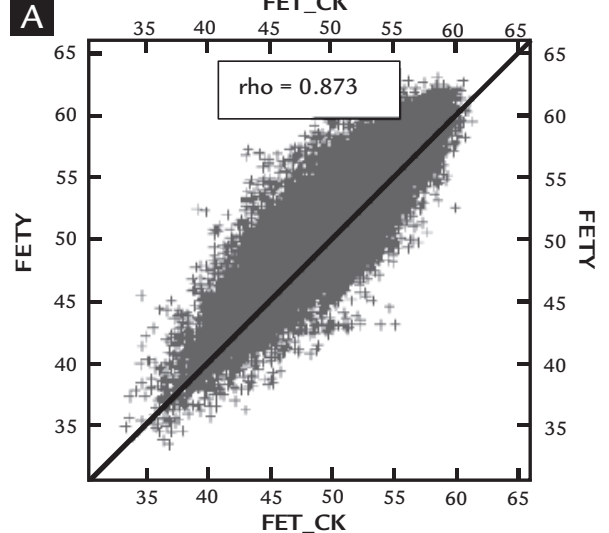

B

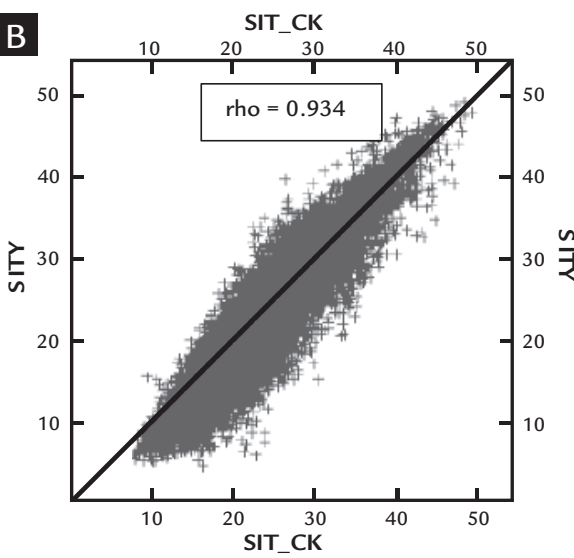

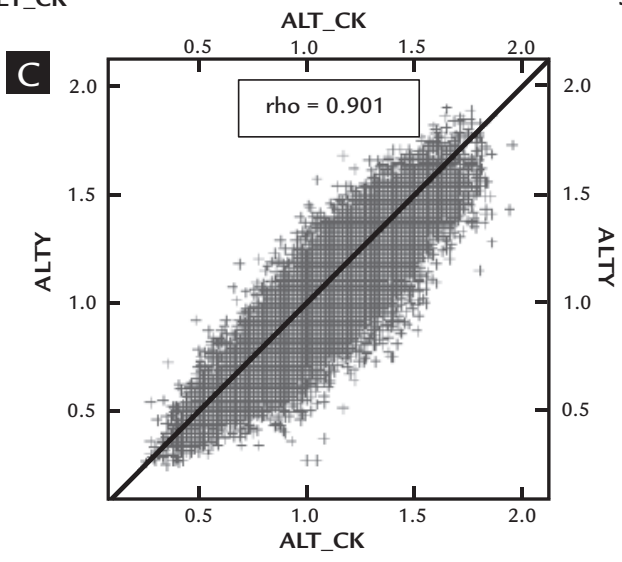

Tabela 3

Média dos dados originais desagrupados, obtida através das estimativas obtidas por cokrigagem das razões logarítmicas aditivas (alr) (CK alr) e também obtida através das estimativas obtidas por cokrigagem dos dados originais sem pósprocessar (CK) e média do número de blocos pós-processados.

Figura 4

Diagramas de dispersão dos teores globais de ferro (A), sílica (B) e alumina (C), obtidos por cokrigagem direta dos dados originais (CK) e por cokrigagem das razões logarítmicas aditivas $(Y)$. 
ब

$X(m)$

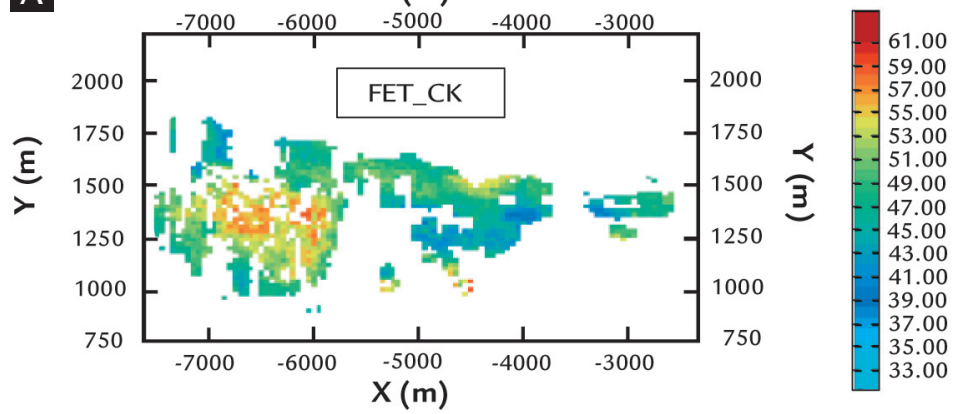

B

Figura 5

Seção $X Y$ dos modelos de blocos do teor de ferro global, para $Z=1345 \mathrm{~m}$, obtidos

por (A) cokrigagem direta dos dados

originais, (B) cokrigagem das razões logarítmicas aditivas (alr). Apresenta-se, ainda, dados originais mais próximos à seção considerada.
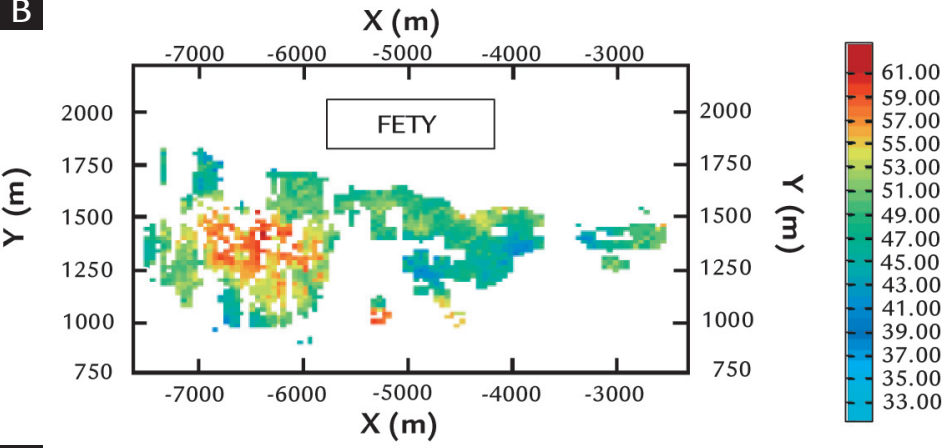

C $X(m)$

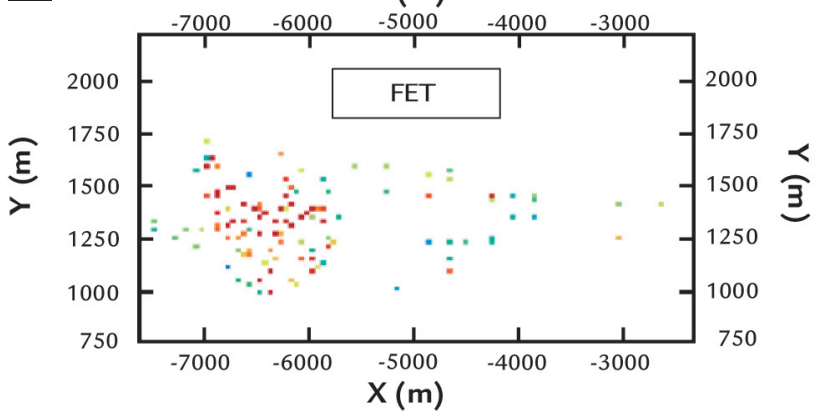
$-56.00$ $-51.00$ 46.00 $-41.00$ 36.00 31.00 26.00
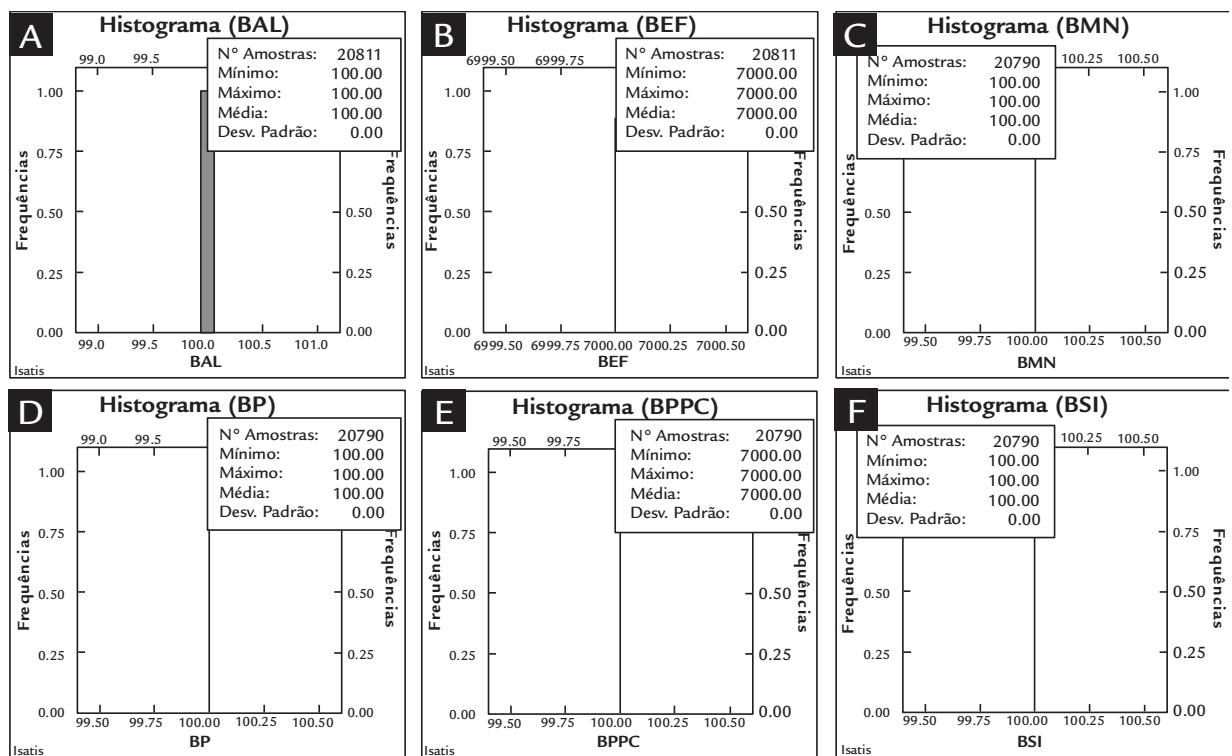

Figura 6

Histograma das somas consideradas, obtida a partir das estimativas para os balanços de massa da alumina BAL (A), ferro BFE (B), manganês BMN (C), fósforo $B P(D)$, perdas por calcinação BPPC (E), sílica BSI (F) e massa BW $(G)$.

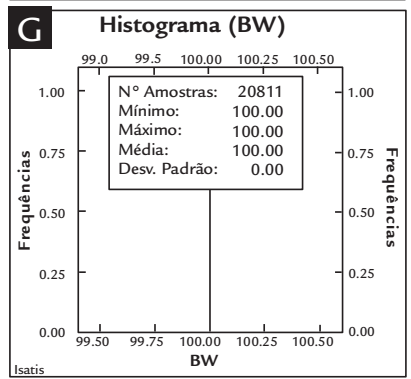



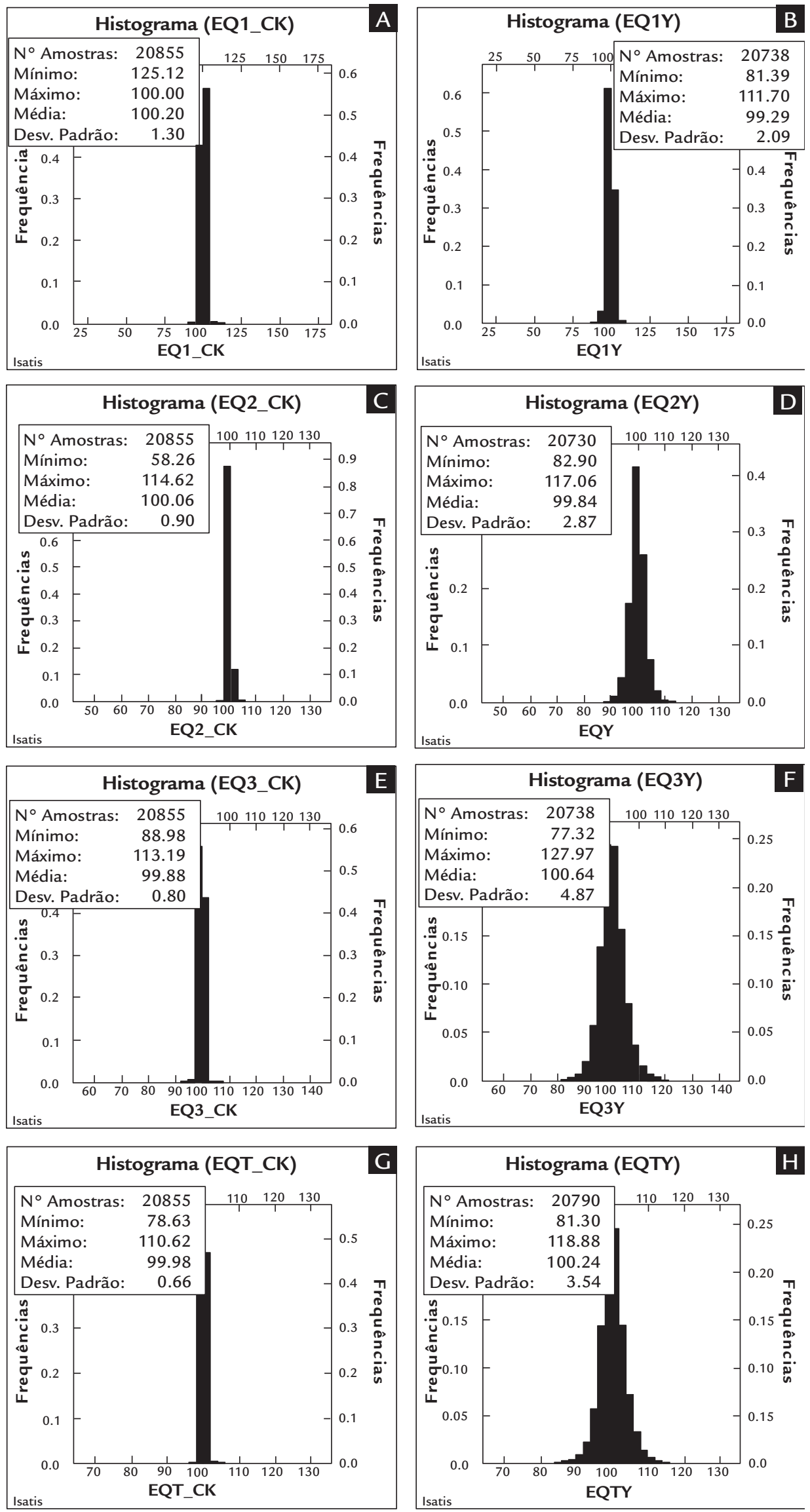

\section{Conclusões}

As variáveis regionalizadas provenientes dos depósitos de minério de ferro, entre outros minérios, estão relacionadas por balanços de massa entre espécies químicas e partições granulométricas, constituindo dados composicionais. $\mathrm{O}$ espaço
Figura 7

Histograma das somas correspondentes aos balanços de massa entre espécies, para cada fração e o global, obtidos a partir das estimativas determinadas por cokrigagem dos dados originais (CK) e das razões logarítmicas aditivas $(Y)$ (fração 1 (A) e (B), fração 2 (C) e (D), fração $3(E)$ e $(F)$ e global $(G)$ e $(H)$, respectivamente). amostral desse tipo de dados é um espaço amostral restrito no qual a soma constante é satisfeita. 
Nesses casos, a soma constante faz com que as correlações espaciais sejam espúrias e, portanto, a sua consideração é incorreta. Ao mesmo tempo, a soma fechada induz uma condição de viés negativo, que leva a estimativas negativas ou fora do intervalo original dos dados.

A cokrigagem de razões logarítmicas aditivas (alr), desenvolvida para lidar com dados composicionais, faz com que todas as estimativas estejam no intervalo original das amostras, sem valores negativos, e faz com que as somas consideradas sejam satisfeitas para a totalidade das estimativas, ficando restringidas no espa- ço amostral original.

Os resultados obtidos reproduzem, adequadamente, as médias globais e locais e provêm mapas das estimativas compatíveis com os dados originais, sem necessidade de pós-processamento.

No caso da cokrigagem direta dos dados originais, não é possível predizer a quantidade de estimativas fora do intervalo original ou negativas. $\mathrm{O}$ fechamento das somas é forçado, deixando uma variável fora do sistema de cokrigagem, como nesse caso, ou, também, pode-se distribuir o erro ou utilizar modelos de corregionalização intrínseca.
Nesse estudo de caso, os resultados obtidos por cokrigagem direta dos dados originais apresentam-se adequados após a substituição dos valores negativos por médias locais.

Para trabalhos futuros, pode se propor a utilização das razoes logarítmicas isométricas $(i l r)$, que preservam melhor a métrica do espaço amostral dos dados composicionais (o D-simplex). No entanto, a transformação em razoes logarítmicas aditivas $(a l r)$ fornece resultados adequados, sendo muito mais simples na sua implementação.

\section{Referências bibliográficas}

AITCHISON, J. A new approach to null correlations of proportions. Mathematical Geology, v.13, n.2, p. 175-189, 1981.

CHILÈS, J. P. \& DELFINER, P. Geostatistics: modelling spatial uncertainty. Nova Iorque: Wiley-Interscience, 1999. 695p.

EGOZCUE, J. J. et al. Isometric log-ratio transformations for compositional data analysis. Mathematical Geology, v. 35, n. 3, p. 279-300.

GOOVAERTS, P. Geostatistics for natural resources evaluation. Nova Iorque: Oxford University Press, 1997. 483p.

ISAAKS, E. H., SRIVASTAVA, R. M. An introduction to applied geostatistics. Nova Iorque: Oxford University Press, 1989. 561 p.

MARECHAL, A. Cokrigeage et regression em correlation intrinsique. Fontainebleau: Centre de Geostatistique de Fontainebleau, 1970. 40p.

MATHERON, G. Principles of geostatistics. Economic Geology, v. 58, p. 1246-1266, 1964.

MUNKES, J. R. Elements of algebraic topology. simplices. Nova Iorque: Perseus Books, 1993. 464 p.

PAWLOWSKY-GLAHN, V., EGOZCUE, J. J. BLU Estimators and Compositional Data. Mathematical Geology, v. 34, n. 3, p. 259-274, 2002.

PAWLOWSKY-GLAHN, V., EGOZCUE, J. J. Geometric approach to statistical analysis o the simplex. Stochastic-Environmental Research and Risk Assesment (SERRA), v. 15, n. 5, p. 384-398, 2001.

PAWLOWSKY-GLAHN, V., OLEA R. Geostatistical analysis of compositional data. Nova Iorque: Oxford University Press, 2004. 181p.

PEARSON, K. Mathematical contributions to the theory of evolution on a form of spurious correlation which may arise when indices are used in the measure of organs. ROYAL SOCIETY OF LONDON, 60, 1897. Anais... p. 489-502.

TOLOSANA-DELGADO, R. Geostatistics for constrained variables: positive data, compositions and probabilities. Application to environmental hazard monitoring. Espanha: Universidade de Girona, 2006. (Tese de Doutorado).

WACKERNAGEL, H. Multivariate geostatistics. An introduction with applications. Berlin: Srpinger Verlag, 1994. 275p.

Artigo recebido em 26 de junho de 2011. Aprovado em 15 de outubro de 2011. 